\title{
Remark on the quasilocal calculation of tidal heating: Energy transfer through the quasilocal surface
}

\author{
Albert Huber $\odot^{*}$ \\ Department Applied Mathematics and Physics, UAS Technikum Wien, \\ Höchstädtplatz 6, 1200 Vienna, Austria
}

(Received 3 May 2021; accepted 19 November 2021; published 4 January 2022)

\begin{abstract}
In this paper, using the quasilocal formalism of Brown and York, the flow of energy through a closed surface containing a gravitating physical system is calculated in a way that augments earlier results on the subject by Booth and Creighton. To this end, by performing a variation of the total gravitational Hamiltonian (bulk plus boundary part), it is shown that associated tidal heating and deformation effects generally are larger than expected. This is because the aforementioned variation leads to previously unrecognized correction terms, including a bulk-to-boundary inflow term that does not appear in the original calculation of the time derivative of the Brown-York energy and leads to corrective extensions of Einstein's quadrupole formula in the large sphere limit.
\end{abstract}

DOI: 10.1103/PhysRevD.105.024011

\section{INTRODUCTION}

The influence of tidal deformation and heating effects on a nearly isolated gravitating systems due to external fields was successfully treated and described by Booth and Creighton in [1]. Instead of using pseudotensorial techniques, which were more popular at the time [2-4], the authors used the Brown-York quasilocal formalism to address the problem. Specifically, in order to describe the interaction of a spatially and temporally bounded gravitating system with an external tidal field, they performed a variation of the boundary part of the total gravitational Hamiltonian, which allowed them to calculate energy and momentum fluxes on a quasilocal surface and thus determine the tidal work done by an external gravitational field in the so-called "buffer zone". Taking advantage of the fact that the associated quasilocal energy expression coincides with the Arnowitt-Deser-Misner energy at spatial infinity [5-7] and the Trautman-BondiSachs energy at null infinity [8-11], the authors provided two interesting applications of their formulas.

First, the authors calculated the energy flux due to gravitational waves through a quasilocal surface in the wave zone (near infinity) to obtain the correct mass loss for a system radiating gravitational waves.

\footnotetext{
*hubera@technikum-wien.at
}

Published by the American Physical Society under the terms of the Creative Commons Attribution 4.0 International license. Further distribution of this work must maintain attribution to the author(s) and the published article's title, journal citation, and DOI.
Second, the authors calculated the tidal heating of a selfgravitating body interacting with an external tidal field in the local asymptotic reference frame of the body. While this effect typically occurs for arbitrary pairs of bodies moving around each other in noncircular orbits, the example discussed in [1] is one that is familiar from the solar system, namely the tidal deformation and associated tidal heating of the Gallilean moon Io by Jupiter, which is generally held responsible for the strong volcanic activity on Jupiter's satellite.

As will be shown in the second section of this work (after a brief overview of the essentials of the considered quasilocal geometric framework in the first section), particularly with respect to the latter application, the analysis of Booth and Creighton can be extended in one particular respect: The mass-energy transfer through the quasilocal surface can alternatively be calculated by varying the total gravitational Hamiltonian (bulk plus boundary parts), which, in contrast to the results originally obtained by the authors, leads to the emergence of correction terms; that is, a confined stress-energy term and a bulk-toboundary inflow term that combines the dynamical degrees of freedom of the bulk with those of the boundary. Although this does not change the validity of the original results of Booth and Creighton (the authors considered a case in which the correction terms become zero), it is nevertheless argued in the present work that the latter term must be taken into account in the quasilocal calculation of more violent tidal deformations and heating effects than those treated in [1], especially in cases of tidal deformations of celestial bodies revolving in circular motion around each other, where there is considerable mass and/or radiation transfer through the quasilocal surface (possibly due to the 
collapse of one of the bodies). To illustrate this, a recap of Booth and Creighton's results on the tidal heating of isolated bodies is given in the third section of this work, followed by a discussion of the extent to which these results are insufficient to describe fluctuations of the interior field of tidally deformed bodies that lead to non-negligible backreactions to the exterior fields of these objects. In this context, specific ideas from the theory of extended irreversible thermodynamics are used as the basis for the discussion. Apart from that, another comparatively simple geometrical example is discussed, namely the mass-energy inflow through the quasilocal surface of a spatially and temporally bounded gravitating system, caused by gravitoelectromagnetic (GEM) fields. Using this example, it is argued that corrections to the time derivative of the BrownYork mass occur and lead to corrections to the Einstein quadrupole formula in the large sphere limit; corrections that should find application in Einstein-Hilbert gravity not only in the special cases discussed in this paper, but in many cases of interest, including the description of, for example, tidal deformation and tidal heating effects caused by accretion phenomena or merger processes in relativistic $N$-body systems.

\section{GEOMETRIC SETTING AND QUASILOCAL ACTION}

As a prerequisite for later considerations, this section provides a brief introduction to the quasilocal framework discussed in [1,12] and reviews some of the important aspects of the underlying geometric model. To that end, a spacetime $(\mathcal{M}, g)$ with manifold $\mathcal{M}=M \cup \partial M$ shall be considered, which is foliated by a family of $t=$ consthypersurfaces $\left\{\Sigma_{t}\right\}$. Evolving continuously between two fixed instants of time $\Sigma_{1}$ and $\Sigma_{2}$, this spacetime shall be assumed to be temporally and spatially bounded. That is to say, it shall be assumed to be bounded by a timelikehypersurface $\mathcal{B}$ in such way that there holds $\partial M=$ $\Sigma_{1} \cup \Sigma_{2} \cup \mathcal{B}$, where none of the embedded boundary parts shall strictly be assumed to be smooth; however, the timelike portion $\mathcal{B}$ shall be assumed to be connected for the sake of simplicity. Moreover, the timelike boundary part shall be assumed to be foliated by a collection of two-surfaces $\left\{\Omega_{t}\right\}$ such that $\mathcal{B}=\left\{\cup_{t} \Omega_{t}: t_{1} \leq t \leq t_{2}\right\}$. As a result of the fixings made, it is ensured that both spatial boundary parts $\Sigma_{1}$ and $\Sigma_{2}$ are given in such a way that they contain a pair of two-surfaces $\Omega_{1}$ and $\Omega_{2}$, respectively, which bifurcate $\Sigma_{1}$ and $\Sigma_{2}$ locally.

The world tube thus obtained divides spacetime into two distinct parts; an interior part $\mathcal{M}$ and an exterior part $\overline{\mathcal{M}}$, in such a way that the interior part $\mathcal{M}$ is contained in the Lorentzian manifold $\overline{\mathcal{M}}$ of an associated ambient spacetime $(\overline{\mathcal{M}}, \bar{g})$ such that $\mathcal{M} \subset \overline{\mathcal{M}}$ and hence $\Sigma_{1}, \Sigma_{2}, \mathcal{B} \subset \overline{\mathcal{M}}$.

Given this specific geometric setting, one can now select a time evolution vector field $t^{a}$ which can be decomposed in components perpendicular and parallel to the leaves of the spacelike foliation of the local spacetime $(\mathcal{M}, g)$. This means that $t^{a}$ can be written-everywhere except at the timelike boundary $\mathcal{B}$-in the form $t^{a}=N n^{a}+N^{a}$, where $N$ is the lapse function, $N^{a}$ is the shift vector field and $n^{a}$ represents a forward-pointing timelike unit vector field orthogonal to the folia of $(\mathcal{M}, g)$. At the timelike boundary $\mathcal{B}$, however, the same vector field must be decomposed alternatively in the form $t^{a}=\mathcal{N} v^{a}+\mathcal{N}^{a}$, where $\mathcal{N}$ is the boundary lapse function, $\mathcal{N}^{a}$ is the boundary shift vector field and $v^{a}$ is a forward-pointing timelike unit vector field being orthogonal to $\Omega_{t}$ and tangent to $\mathcal{B}$. By the selection of a time-flow vector field, a time parameter $t$ can now be fixed by solving the relation $L_{t} t=1$ (according to which $L_{t}$ represents the Lie derivative along $t^{a}$ ).

Next, it may be assumed that there exists an outwardpointing spacelike unit normal to $\mathcal{B}$, henceforth denoted by $u^{a}$, which is usually nonorthogonal to the timelike generator $n^{a}$, but orthogonal to another timelike unit vector field $v^{a}$ that is tangent to $\mathcal{B}$. At the same time, there is a spacelike vector field $s^{a}$ which is orthogonal to the timelike generator $n^{a}$. Due to the nonorthogonality of the two vector fields $n^{a}$ and $u^{a}$, there must exist a scalar field $\eta \equiv u_{a} n^{a}$, which vanishes only for the special case of a timelike boundary which is exactly orthogonal to all leaves of its spacelike foliation. This scalar field also occurs in the relation $s_{a} v^{a} \equiv-\eta$ and in the decomposition relations $v^{a}=$ $\lambda\left(n^{a}-\eta u^{a}\right), \quad s^{a}=\lambda\left(u^{a}+\eta n^{a}\right)$ and $n^{a}=\lambda\left(v^{a}+\eta s^{a}\right)$, $u^{a}=\lambda\left(s^{a}-\eta v^{a}\right)$, whose validity follows directly from that of the normalization conditions $u^{a} u_{a}=s^{a} s_{a}=$ $-n^{a} n_{a}=-v^{a} v_{a}=1$, where $\lambda=\frac{1}{\sqrt{1+\eta^{2}}}$ is a boost parameter. This boost parameter also occurs in the relation $\mathcal{N}=\lambda N$ between the standard lapse function $N$ and the boundary lapse function $\mathcal{N}$, which occur in the volume elements of spacetime and that of the embedded spacelike boundary hypersurface, i.e., $\sqrt{-g}=N \sqrt{h}$ and $\sqrt{-\gamma}=\mathcal{N} \sqrt{q}$. These relations, in turn, apply with respect to the determinant of the four-metric $g_{a b}$ of $(\mathcal{M}, g)$, its induced three-metrics $h_{a b}=g_{a b}+n_{a} n_{b}$ on $\Sigma_{t}$ and $\gamma_{a b}=$ $g_{a b}-u_{a} u_{b}$ on $\mathcal{B}$ and the two-metric $q_{a b}=g_{a b}+n_{a} n_{b}-$ $s_{a} s_{b}=g_{a b}-u_{a} u_{b}+v_{a} v_{b}$ that is induced by $g_{a b}$ on $\Omega_{t}$.

Ultimately, as a byproduct of considering all these definitions, Hayward's quasilocal action [13]

$$
\begin{aligned}
S_{G}[g]= & \frac{1}{16 \pi}\left(\int_{M} R \omega_{g}+2 \int_{\Sigma_{1}}^{\Sigma_{2}} K \omega_{h}\right. \\
& \left.+2 \int_{\mathcal{B}} \Theta \omega_{\gamma}+2 \int_{\Omega_{1}}^{\Omega_{2}} \sinh ^{-1} \eta \omega_{q}\right),
\end{aligned}
$$

can finally be set up, where, in this context, $R=g^{a b} R_{a b}$ denotes the Ricci scalar of the spacetime $(\mathcal{M}, g)$, and $K=$ $h^{a b} K_{a b}$ and $\Theta=\gamma^{a b} \Theta_{a b}$ term the scalar extrinsic curvatures 
of the spacelike folia $\Sigma_{t}$ and the timelike boundary $\mathcal{B}$ of $(\mathcal{M}, g)$. Moreover, as can be seen, the different volume elements $\omega_{g}:=\sqrt{-g} d^{4} x, \omega_{h}:=\sqrt{h} d^{3} x, \omega_{\gamma}:=\sqrt{-\gamma} d t d^{2} x$, and $\omega_{q}:=\sqrt{q} d^{2} x$ enter the definition of this quasilocal action.

Following closely the steps taken in [6,12], one finds that Hayward's action can be rewritten in a slightly different form. More specfically, using the decomposition relation $R={ }^{(3)} R+K_{a b} K^{a b}-K^{2}+2 \nabla_{b}\left(K n^{b}-a^{b}\right)$ for the Ricci scalar, the Hamiltonian and momentum constraints $\mathcal{H}=$ $\frac{8 \pi}{\sqrt{h}}\left(P_{a b} P^{a b}-\frac{1}{2} P^{2}\right)-\frac{\sqrt{h}}{16 \pi}{ }^{(3)} R$ and $\mathcal{H}_{a}=-2 D_{b} P^{b}{ }_{a}$, which are defined with respect to the fields $P^{a b}=\frac{\sqrt{h}}{16 \pi}\left(K^{a b}-\right.$ $\left.h^{a b} K\right), K_{a b}=\frac{1}{2 N}\left(\dot{h}_{a b}-2 D_{(a} N_{b)}\right)$, and $\frac{\sqrt{-g}}{16 \pi}\left(K_{a b} K^{a b}-K^{2}\right)=$ $P_{a b} \dot{h}^{a b}-\frac{8 \pi N}{\sqrt{h}}\left(P_{a b} P^{a b}-\frac{1}{2} P^{2}\right)-2 P_{a b} D^{a} N^{b}$, as well as the identities $k=\lambda\left(\Theta+\eta K-a_{b} u^{b}+\lambda(v \nabla) \eta\right), N k-\left(K_{a b}-\right.$ $\left.K h_{a b}\right) N^{a} s^{b}=\mathcal{N} \mathfrak{K}-\lambda(\mathcal{N} \nabla) \eta-(\mathcal{N} \nabla) v_{a} u^{a}$, in relation to which $\mathfrak{K}=q^{a b} \mathfrak{\mathfrak { K }}_{a b}=q^{a b} \nabla_{a} u_{b}$ represents the extrinsic curvature of the spacelike intersection surfaces $\Omega_{t}$ formed in relation to $u^{a}$, one finds that Hayward's action can be rewritten in the form

$$
\begin{aligned}
S_{G}[g]= & \int d t \int_{\Sigma_{t}} d^{3} x\left[P_{a b} h^{a b}-N \mathcal{H}-\mathcal{H}_{a} N^{a}\right] \\
& +\frac{1}{8 \pi} \int d t \int_{\Omega_{t}} \dot{\omega}_{q} \sinh ^{-1} \eta \\
& +\int d t \int_{\Omega_{t}} d^{2} x\left[\mathcal{N} \mathfrak{h}+\mathfrak{h}_{a} \mathcal{N}^{a}\right],
\end{aligned}
$$

where $\mathfrak{h}=\frac{\sqrt{q}}{8 \pi} \mathfrak{K}, \mathfrak{h}_{a}=-\frac{\sqrt{q}}{8 \pi} u^{c} q^{b}{ }_{a} \nabla_{b} v_{c}$ and the overdot denotes the Lie derivative with respect to $t^{a}$.

As a result of these conversions of Hayward's quasilocal action, the generalized gravitational Hamiltonian

$$
H=\int_{\Sigma_{t}}\left(N \mathcal{H}+\mathcal{H}_{a} N^{a}\right) d^{3} x-\int_{\Omega_{t}}\left(\mathcal{N} \mathfrak{h}+\mathfrak{h}_{a} \mathcal{N}^{a}\right) d^{2} x
$$

is obtained, whose precise form was first determined by Brown and York in the course of their field-theoretical generalization of Hamilton-Jacobi theory [6]. Given with respect to the canonical variables $\left(h^{a b}, P_{a b}\right)$ and $\left(\sqrt{q}, \sinh ^{-1} \eta\right)$, this generalized gravitational Hamiltonian provides a feasible quasilocal description of the total amount of energy momentum of a gravitational field with spatially and temporally finite dimensions, which represents an alternative to more traditional pseudotensorial descriptions of the subject. ${ }^{1}$

\footnotetext{
${ }^{1}$ For a detailed review, see for instance [14].
}

An important characteristic of the given generalized Hamiltonian is the fact that it splits up into a bulk part $H_{\text {Bulk }}=\int_{\Sigma_{t}}\left(N \mathcal{H}+\mathcal{H}_{a} N^{a}\right) d^{3} x$ and a boundary part $H_{\text {Boundary }}=-\int_{\Omega_{t}}^{t}\left(\mathcal{N} \mathfrak{h}+\mathfrak{h}_{a} \mathcal{N}^{a}\right) d^{2} x$, where, as already indicated, the boundary part $H_{\text {Boundary }}$ coincides in an asymptotically flat spacetime in the so-called large sphere limit with well-known candidates for the total quasilocal stress-energy momentum of the gravitational field itselfwith that of Arnowitt, Deser, and Misner at spacelike infinity and that of Bondi and Sachs at null infinity.

Given the exact form of the quasilocal gravitational Hamiltonian (3), the quasilocal charge

$$
E=\int_{\Sigma_{t}}\left(N \varepsilon-p_{a} N^{a}\right) \omega_{h}-\int_{\Omega_{t}}\left(\mathcal{N} e-j_{a} \mathcal{N}^{a}\right) \omega_{q},
$$

can be derived by using Einstein's equations

$$
G_{a b}=8 \pi T_{a b}
$$

As may be noted, the quasilocal charge obtained again consists of two parts; a bulk part $E_{\mathrm{Bulk}}=\int_{\Sigma_{t}}\left(N \varepsilon-p_{a} N^{a}\right) \omega_{h}$ and a boundary part $E_{\text {Boundary }}=-\int_{\Omega_{t}}\left(\mathcal{N}^{t}-j_{a} \mathcal{N}^{a}\right) \omega_{q}$.

This all is of significance in that the results obtained so far form the starting point for Booth and Creighton's quasilocal calculation of tidal heating, which will be briefly discussed in the third and final section of this paper. However, for the analysis of the results of the now upcoming second section of this work, it is important to note that the authors considered only the boundary part of expression (4) as the basis for their quasilocal calculation of tidal heating. More specifically, an important step in their calculation was to determine the temporal variation of said boundary part, for which they obtained the result

$$
\frac{d W}{d t}=\int_{\Omega_{t}}\left(-\dot{\mathcal{N}} e+j_{a} \dot{\mathcal{N}}^{a}+\frac{\mathcal{N}}{2} s^{a b} \dot{q}_{a b}\right) \omega_{q}
$$

As will be shown in the next section, the calculation yields a different result when the full quasilocal expression (4) (and only its boundary part) is considered. Specifically, it is shown that the temporal variation of the quasilocal charge (4) leads to correction terms that do not appear in the original calculation of Booth and Creighton, but must typically be included when considering nonvanishing stress-energy tensors. As is argued later in the third and final section of this work, this may prove to be of relevance not only for the determination of tidal heating effects, but also for other applications in the quasilocal approach to general relativity. 


\section{GRAVITATIONAL HAMILTONIAN AND QUASILOCAL ENERGY FLUX}

With the results of the previous section at hand, the next step will be to derive the exact form of the correction terms mentioned in the introduction. For this purpose, a temporal variation of the total functional $E$ (bulk plus boundary) shall be calculated in the following by Lie dragging it along the flow of the time evolution vector field $t^{a}$. As a basis for doing this, the decomposition relations $T_{a b}=\varepsilon n_{a} n_{b}+$ $n_{a} p_{b}+p_{a} n_{b}+S_{a b}$ and $\tau_{a b}=e v_{a} v_{b}+v_{a} j_{b}+j_{a} v_{b}+s_{a b}$ will be used, whose validity implies that $T_{a b} t^{a} n^{b}=N \varepsilon-$ $p_{a} N^{a}$ and $\tau_{a b} t^{a} v^{b}=\mathcal{N} e-j_{a} \mathcal{N}^{a}$ holds true with respect to any given pair of (totally general) stress-energy and surface stress-energy tensors $T_{a b}$ and $\tau_{a b}$. As may be noted in this context, the fields $p_{a}=h_{a}{ }^{c} T_{c b} n^{b}$ and $j_{a}=q_{a}{ }^{c} \tau_{c b} v^{b}$ represent the mass-energy and surface mass-energy fluxes and $S_{a b}=h_{a}{ }^{c} h_{b}{ }^{d} T_{c d}$ and $s_{a b}=q_{a}{ }^{c} q_{b}{ }^{d} \tau_{c d}$ represent the stress and surface stress tensors of matter fields located within the bounded spacetime region $\mathcal{M} \subset \overline{\mathcal{M}}$.

Assuming that $\mathfrak{\Omega}_{t}$ denotes the induced Lie derivative at $\Sigma_{t}$ pointing along $t^{a}$, it proves to be instrumental for the calculation of the time derivative of $E$ to note that

$$
\begin{aligned}
\mathfrak{\Omega}_{t}\left[\omega_{h}\left(N \varepsilon-p_{a} N^{a}\right)\right]= & \omega_{h} \mathfrak{Q}_{t}\left(N \varepsilon-p_{a} N^{a}\right) \\
& +\omega_{h}\left(N K+D_{b} N^{b}\right)\left(N \varepsilon-p_{a} N^{a}\right),
\end{aligned}
$$

holds true with respect to any spacetime $(\mathcal{M}, g)$ whose geometric structure permits consideration of the quasilocal approach described in the very first section. Moreover, it proves beneficial to heed another observation; the local conservation laws $\nabla_{a} T^{a}{ }_{c} \cdot N n^{c}=\nabla_{a} T^{a}{ }_{c} \cdot N^{c}=0$, in combination with the decompositions of $T_{a b}$ and $\tau_{a b}$ mentioned above, lead to the generalized continuity and contracted generalized Euler equations

$\mathfrak{\Omega}_{t} \varepsilon=(N D) \varepsilon-\varepsilon N K-N D_{a} p^{a}-2(p D) N-N S_{a b} K^{a b}$,

and

$$
\begin{aligned}
& \mathfrak{\Omega}_{t} p_{a} \cdot N^{a}=(N D)\left(p_{a} N^{a}\right)-N D_{b} S^{b}{ }_{a} N^{a}-S^{b}{ }_{a} N^{a} D_{b} N \\
& -N K p_{a} N^{a}-\varepsilon(N D) N \text {; }
\end{aligned}
$$

equations that were first published (though in terms of some slightly different conventions) many years ago in [15]. For notational clarification, it may be noted here that the definitions $(N D):=N^{a} D_{a}$ and $(p D):=p^{a} D_{a}$ have been used in the present context.

By suitably combining these relations and applying the inverse Leibniz rule with the idea of producing a total divergence term, one obtains the result

$N \boldsymbol{\Omega}_{t} \varepsilon-\boldsymbol{\Omega}_{t} p_{a} \cdot N^{a}=D_{a}\left[\left(N \varepsilon-p_{b} N^{b}\right) N^{a}-N^{2} p^{a}+N S^{a}{ }_{b} N^{b}\right]-\left(N K+D_{a} N^{a}\right)\left(N \varepsilon-p_{b} N^{b}\right)-N S_{a b}\left(N K^{a b}+D^{(a} N^{b)}\right)$.

Therefore, in further consequence, one finds

$$
\mathfrak{Q}_{t}\left[\omega_{h}\left(N \varepsilon-p_{a} N^{a}\right)\right]=\omega_{h}\left(\mathfrak{Q}_{t} N \varepsilon-p_{a} \mathfrak{Q}_{t} N^{a}+\frac{N}{2} S_{a b} \mathfrak{Q}_{t} h^{a b}\right)+\omega_{h} D_{a}\left[\left(N \varepsilon-p_{b} N^{b}\right) N^{a}-N^{2} p^{a}+N S^{a}{ }_{b} N^{b}\right] .
$$

By applying Gauss' law in the next step and using the constraint relation $\mathcal{D}_{a} \tau^{a}{ }_{b}=\pi^{a}=\gamma^{a}{ }_{b} T_{a c} u^{c}$, in respect to which $\mathcal{D}_{a} \tau^{a}{ }_{b}=\gamma_{b}{ }_{b} \gamma_{c}{ }_{c} \nabla_{e} \tau_{d}^{c}$ applies, the temporal variation of the functional $E$ can now straightforwardly be calculated, leading to the result

$$
\frac{d E}{d t}=\int_{\Sigma_{t}}\left(\dot{N} \varepsilon-p_{a} \dot{N}^{a}+\frac{N}{2} S_{a b} \dot{h}^{a b}\right) \omega_{h}-\int_{\Omega_{t}}\left(\dot{\mathcal{N}} e-j_{a} \dot{\mathcal{N}}^{a}+\frac{\mathcal{N}}{2} s_{a b} \dot{q}^{a b}-\mathfrak{X}\right) \omega_{q} .
$$

Here, it should be noted that to obtain this very integral expression, the definitions $s_{a b}=q_{a}{ }^{c} q_{b}{ }^{d} \tau_{c d}, b^{a} \equiv(v \nabla) v^{a}$, $\dot{N}=\mathfrak{\Omega}_{t} N, \dot{N}^{a}=\mathfrak{\Omega}_{t} N^{a}, \dot{h}^{a b}=\mathfrak{\Omega}_{t} h^{a b}$ as well as $\dot{\mathcal{N}}=\mathcal{L}_{t} \mathcal{N}$, $\dot{\mathcal{N}}^{a}=\mathcal{L}_{t} \mathcal{N}^{a}, \dot{q}^{a b}=\mathcal{L}_{t} q^{a b}$ have been used, where $\mathcal{L}_{t}$ denotes the induced Lie derivative at $\Omega_{t}$ pointing along $t^{a}$.

The integrand $\mathfrak{X}$ occurring in the boundary part of Eq. (12) consists of two parts, i.e.,

$$
\mathfrak{X}=\mathfrak{X}_{1}+\mathfrak{X}_{2},
$$

where

$$
\mathfrak{X}_{1}=\mathcal{N}^{2} \pi_{a} v^{a}+\mathcal{N} \pi_{a} \mathcal{N}^{a}
$$

represents the confined stress-energy term and

$$
\begin{aligned}
\mathfrak{X}_{2}= & -\frac{\eta}{\lambda} \mathcal{N}^{2} \varepsilon+\eta \mathcal{N}^{2} p_{a} v^{a}+\eta \mathcal{N} p_{a} \mathcal{N}^{a} \\
& -\frac{\mathcal{N}^{2}}{\lambda} p_{a} u^{a}+\lambda \mathcal{N} S_{a b} v^{a} u^{b}+\lambda S_{a b} \mathcal{N}^{a} u^{b}
\end{aligned}
$$

represents a bulk-to-boundary inflow term, as may be concluded from the fact that the bulk expressions $\varepsilon, p_{a}$, 
and $S_{a b}$ enter into its definition. ${ }^{2}$ For the sake of clarification, it may be noted here that the relations $N_{a} s^{a}=-\eta \mathcal{N}$, $N \varepsilon-p_{a} N^{a}=\frac{\mathcal{N}}{\lambda} \varepsilon-\mathcal{N} p_{a} v^{a}-p_{a} \mathcal{N}^{a}, N^{2} p_{a} s^{a}=\frac{\mathcal{N}^{2}}{\lambda} p_{a} u^{a}$, and $S_{a b} s^{a} N^{b}=\lambda \mathcal{N} S_{a b} v^{a} u^{b}+\lambda S_{a b} \mathcal{N}^{a} u^{b}$ have been used in order to obtain this particular form of equation (15). The corresponding integral term that occurs in relation (12), which results from converting the total divergence appearing in Eq. (11), is equal to the net flux of quasilocal bulk energy passing through $\Omega_{t}$ being transferred from the gravitating physical system under consideration (through the spatial boundary) to its environment.

Upon closer inspection of the full boundary part of integral expression (12), it can further be observed that said part of the expression (on account of its physical units) can be interpreted as a power functional of the form $\mathcal{P}=\int_{\Omega_{t}} \mathcal{I} \omega_{q}$, where the corresponding integrand reads

$$
\mathcal{I}=\dot{\mathcal{N}} e-j_{a} \dot{\mathcal{N}}^{a}+\frac{\mathcal{N}}{2} s_{a b} \dot{q}^{a b}-\mathfrak{X}
$$

However, such an interpretation only truly makes sense if electromagnetic and/or gravitational radiation passes through the quasilocal surface $\Omega_{t}$; a case in which the quantity $\mathcal{I}$ typically can be identified as the intensity of the total radiant energy escaping from the system into the environment. But, as shall be discussed in greater detail in the next section, where applications will be considered, the integral $\int_{\Omega_{t}} \mathfrak{X}_{2} \omega_{q}$ cannot be neglected.

Before that, however, Eq. (12) shall first be considered from a different angle in order to also illuminate the geometric aspect of the chosen approach. For this purpose, one may rewrite (12) using the field equations of the theory, which yields the result

$$
\begin{aligned}
\frac{d H}{d t} & =\int_{\Sigma_{t}}\left(\dot{N} \mathcal{H}-\mathcal{H}_{a} \dot{N}^{a}+\frac{N}{2} \mathcal{Q}_{a b} \dot{h}^{a b}\right) d^{3} x \\
& -\int_{\Omega_{t}}\left(\dot{\mathcal{N}} \mathfrak{h}-\mathfrak{h}_{a} \dot{\mathcal{N}}^{a}+\frac{\mathcal{N}}{2} \mathfrak{Q}_{a b} \dot{q}^{a b}-\sqrt{q} \mathfrak{X}\right) d^{2} x,
\end{aligned}
$$

for the temporal variation of the gravitational Hamiltonian. As may be noted, the definitions

$$
\begin{aligned}
\mathcal{Q}_{a b}= & \frac{\sqrt{h}}{8 \pi}\left\{{ }^{(3)} R_{a b}-2 K_{a c} K_{b}^{c}+K K_{a b}-\frac{1}{N}\left[\dot{K}_{a b}+(N D) K_{a b}+D_{a} D_{b} N\right]\right. \\
& \left.-\frac{1}{2} h_{a b}\left({ }^{(3)} R+K^{2}-K_{c d} K^{c d}-\frac{2}{N}\left[\dot{K}+(N D) K+D_{a} D^{a} N\right]\right)\right\},
\end{aligned}
$$

and

$$
\mathfrak{Q}_{a b}=\frac{\sqrt{q}}{8 \pi}\left(\Re_{a b}-\left(\mathfrak{\Re}-b_{a} u^{a}\right) q_{a b}\right),
$$

have been used in the present context. Moreover, as may also be noted, relation (18) has been used in combination with the constraint equations of the theory to rewrite relations (13) and (14) in the form

$$
\mathfrak{X}_{1}=\mathcal{N}^{2} \mathcal{D}_{a} \tau_{b}^{a} v^{b}+\mathcal{N} \mathcal{D}_{a} \tau_{b}^{a} \mathcal{N}^{a}
$$

and

$$
\begin{aligned}
\mathfrak{X}_{2}= & -\frac{\eta}{\lambda} \mathcal{N}^{2} \mathcal{H}-\frac{1}{2} \eta \mathcal{N}^{2} \mathcal{H}_{a} v^{a}-\frac{1}{2} \eta \mathcal{N} \mathcal{H}_{a} \mathcal{N}^{a} \\
& -\frac{\mathcal{N}^{2}}{2 \lambda} \mathcal{H}_{a} u^{a}+\lambda \mathcal{N} \mathcal{Q}_{a b} v^{a} u^{b}+\lambda \mathcal{Q}_{a b} \mathcal{N}^{a} u^{b},
\end{aligned}
$$

\footnotetext{
${ }^{2}$ Note that the bulk fields $\varepsilon, p_{a}$, and $S_{a b}$ can, of course, be reexpressed on the boundary in terms of associated boundary fields. However, in order to highlight the fact that these fields can be used to interpret $\int_{\Omega_{t}} \mathfrak{X}_{2} \omega_{q}$ as a bulk-to-boundary inflow, the form of $\varepsilon, p_{a}$, and $S_{a b}$ has been intentionally left unchanged at this point.
}

and therefore obtain with (17) a purely geometrical expression that agrees exactly with the time derivative of the quasilocal gravitational Hamiltonian. Here, as may be noted, the use of Einstein's equations (or rather the constraint equations resulting from them) does not prove to be mandatory in this context, but rather practical, since all calculations could also be carried out exclusively with geometric quantities, which, of course, yields exactly the same result in the end.

As may be noted, there is something both astonishing and appealing about results (12) and (17), namely, that the occurring bulk and boundary integral expressions look very similar; only the correction terms prevent an exact similarity. From the structure of (17) it can be generally concluded that a change of the matter content of a spatially and temporally bounded gravitating system always depends on how the individual components of the metric evolve over time. Moreover, it can be concluded that such a change also strongly depends on the structure of the occurring correction terms, which can be interpreted physically as a bounded mass-energy distribution plus a nonvanishing bulk to boundary inflow that combines the dynamical degrees of freedom of the bulk with those of the boundary; even if it is assumed that the considered local 
gravitational field is stationary. It can be expected that pseudotensorial methods lead to similar results, although further studies of such an approach may be in order.

Anyway, to illustrate the physical relevance of the derivations made, some selected applications will now be discussed in the next section, with a particular focus on possibly testable implications of the theory. In particular, two effects will be examined in more detail, namely the loss of quasilocal mass energy due to (i) tidal deformation and heating, and (ii) the emission of gravitational, electromagnetic, and/or GEM radiation. With respect to the first type of effect, as it turns out, the model proves consistent with the results of Booth and Creighton in the sense that the derived correction terms play no role in calculating the loss of quasilocal mass due to tidal heating. The reason for this, as will be shown, is that the authors' approach remains fully valid precisely when the source-free Einstein equations are considered (as done in [1]), whereas it must very well be extended if matter fields are present and thus the nonvacuum field equations apply. However, this is exactly what is to be expected in the presence of tidal effects which lead to perturbations not only of the external but also of the internal field of a body. Something similar is also to be expected, as shall be made clear below, in the second case to be discussed, which is devoted to the combined emission of gravitational and electromagnetic radiation by a gravitating physical system.

\section{TIDAL HEATING, GRAVITOELECTROMAGNETISM, AND FURTHER APPLICATIONS}

In view of the results derived in the previous section, the question naturally arises whether or not the derived correction terms play a role for the quasilocal calculation of tidal heating, and whether or not, therefore, the original results of Booth and Creighton from [1] need to be modified or generalized in some sense. Unsurprisingly, as will be explained below, the authors' approach turns out to be perfectly sound (in terms of the specific assumptions made in their work). However, as shall also be made clear below, said approach needs very well to be generalized in cases where quasilocal methods are to be used to describe more violent tidal deformations and heating effects than those discussed in [1], such as in cases of tidal deformations of celestial bodies in $\mathrm{N}$-body systems that are accompanied by substantial mass and/or radiation transfer through the quasilocal surface, as occur for example when one of the bodies collapses or pairs of bodies merge with each other.

Before discussing mass loss due to such effects, however, it is first necessary to show that Booth and Creighton's results occur as a special case of those derived in the previous section. To show this, it is sufficient to make a single assumption, namely that the considered spacetime $(\mathcal{M}, g)$ is Ricci-flat and therefore the on-shell vacuum field equations

$$
R_{a b}=0,
$$

are satisfied; a requirement that has the effect that the time derivative of the bulk part appearing in (12) becomes zero in the same way as the derived correction terms, which further implies that this relation reduces to the form

$$
\frac{d E}{d t}=-\int_{\Omega_{t}}\left(\dot{\mathcal{N}} e-j_{a} \dot{\mathcal{N}}^{a}+\frac{\mathcal{N}}{2} s_{a b} \dot{q}^{a b}\right) \omega_{q} .
$$

Consequently, however, by utilizing the fact that $\dot{q}^{a b}=$ $-q^{a c} q^{b d} \dot{q}_{c d}$ holds in the given context, one finds that relations (6), (12), and (23) all coincide in the case that the source-free Einstein equations are satisfied, thus making it clear that the results of the work of Booth and Creighton occur as a special case of the results derived in section two of this work. Based on this, it can be further concluded that the temporal variation of the full Hamiltonian (bulk plus boundary) needs to be considered only when matter fields are present and spacetime is not Ricci-flat, while in the opposite case it is sufficient to consider the boundary part of the gravitational Hamiltonian, as was done in [1].

Having clarified this, it shall now be briefly be reviewed how the obtained formula can be used to describe tidal deformation and heating effects. To this end, one may recall that Booth and Creighton used the Hartle-Thorne model $[4,16]$ as a basis for their quasilocal calculations, i.e., a perturbative vacuum model that describes how the external field of a small self-gravitating central body changes under the influence of an external tidal field. This very model deals with a spacetime metric $g_{a b}$ of the form

$$
g_{a b}=\eta_{a b}+e_{a b},
$$

which is given with respect to the flat Minkowski metric $\eta_{a b}$, where $\left|e_{a b}\right| \ll 1$ applies by definition. In rectangular coordinates, the individual components of the perturbation tensor $e_{a b}$ take the form $e_{00} \equiv \phi \equiv-\frac{2 M}{r}-\frac{3 Q_{i k} s^{i} s^{k}}{r}+$ $r^{2} \mathcal{E}_{i k} s^{i} s^{k}, \quad e_{0 j} \equiv A_{j} \equiv-\frac{2}{r} \frac{d Q_{j k}}{d t} s^{k}+\frac{4 r^{3}}{21} \frac{d \mathcal{E}_{j k}}{d t} s^{k}-\frac{10 r^{3}}{21} \frac{d \mathcal{E}_{i k}}{d t} s^{i} s^{k} s_{j}$, and $e_{i j}=\left(\frac{2 M}{r}-\frac{3}{r^{3}} Q_{k l} s^{k} s^{l}+r^{2} \mathcal{E}_{k l} s^{k} s^{l}\right) \delta_{i j}$, where, in this regard, the field $Q_{i k}=\int \varepsilon\left(x_{i} x_{k}-\frac{1}{3} r^{2} \delta_{i k}\right) d^{3} x$ represents the trace-free and symmetric quadrupole moment of the selfgravitating body and $\mathcal{E}_{i k}=\mathcal{R}_{i 0 k 0}$ the electric part of the Weyl tensor, which coincides with the Riemann curvature tensor in the given case. The radial unit vector occurring in this context is given by $s^{i}=\frac{x^{i}}{r}$, where $r$ is the radial distance from the center of the isolated body as measured in its local asymptotic rest frame.

Taking advantage of the gauge ambiguity in setting up a nearly Minkowskian coordinate system and switching to spherical coordinates, it was shown in [1] that the HartleThorne metric (24) can be converted into a slightly different form. More specifically, by using the diffeomorphism 
freedom to make the replacement $e_{a b} \rightarrow e_{a b}+2 \partial_{(a} \xi_{b)}$ with respect to the co-vector field $\xi_{j}=\alpha r^{-2} Q_{j k} s^{k}+$ $\beta r^{3} \mathcal{E}_{j k} s^{k}+\gamma r^{3} \mathcal{E}_{i k} s^{i} s^{k} s_{j}$, where $\alpha, \beta$, and $\gamma$ are free constants of order one, it was shown it the aforementioned work that the rate at which tidal work is performed on an isolated body is given (modulo higher order terms) by the same formula as in the post-Newtonian approximation of the theory, which had previously been derived by using pseudotensorial techniques [2,3]. Using here the shorthand notation $\mathfrak{Y}=\frac{1}{60} \frac{d}{d t}\left[2 a r^{5} \mathcal{E}_{i k} \mathcal{E}^{i k}+2 b \mathcal{E}_{i k} Q^{i k}-c r^{5} Q_{i k} Q^{i k}\right]$ in conjunction with the definitions $a=8 \beta \gamma+4 \gamma^{2}-2 \beta^{2}+4 \gamma-$ $2 \beta-3, b=8 \alpha \gamma-12 \gamma+6 \beta-2 \alpha+3$, and $c=4 \alpha^{2}-12 \alpha-9$, the obtained formula reads

$$
\frac{d E}{d t}=\frac{1}{2} \mathcal{E}_{i k} \frac{d Q^{i k}}{d t}+\mathfrak{Y} .
$$

In this context, the $\frac{1}{2} \mathcal{E}_{i k} \frac{d Q^{i k}}{d t}$-term specifies the irreversible (unrecoverable) part of the work done to deform and heat the system (the dissipated energy is converted into heat), whereas the second $\mathfrak{Y}$-term represents the reversible (recoverable) part of the work being done to increase the potential energy of the system. Regarding these two terms, the former irreversible one turns out to be the one of primary interest, since (i) it is invariant under diffeomorphisms genererated by $\xi_{j}$ (in contrast to the second reversible term) and (ii) it is the same leading-order term that was previously obtained through analogous calculation using pseudotensorial instead of quasilocal methods (which, surprisingly, all lead to the same expression regardless of the choice of energymomentum complex and gauge conditions [2,3]; a situation previously known only from the case of pseudotensors calculated from Kerr-Schild metrics [17-19]). Although not really obvious at the time, this compatibility of results is not very surprising from today's viewpoint in that it has been shown by Chang, Chen, and Nester [20] that any energy-momentum pseudotensor leads to an associated Hamiltonian boundary term, so that it becomes clear that-within the quasilocal Hamiltonian frameworkpseudotensors either give rise to distinct Dirichlet type boundary conditions or to Neumann-type boundary conditions; all of which are algebraic in terms of the metric. Consequently, however, since the tidal heating results obtained in the literature appear to be independent of the choice of boundary conditions, it is hardly surprising that the purely quasilocal calculation of Booth and Creighton leads to very similar results as those of earlier works on the subject that used the pseudotensorial approach. This is not least the case because, in the approximate calculations mentioned, the precise form of the boundary conditions is not essential, because the results obtained by using different boundary conditions deviate from each other only in higher order. Therefore, from a purely mathematical point of view, the good agreement of the results proves to be completely natural.

However, what is striking in this context is that, in contrast to the results of earlier pseudotensorial approaches, the quasilocal results are not only completely general but also coordinate-independent, which is why the results obtained could in principle also be used to determine tidal deformations and heating effects in more sophisticated and physically challenging situations; that is, in situations where not only fluctuations of the external gravitational field of the body are considered, but also changes of the interior field of the self-gravitating body are taken into account. After all, it has to be kept in mind that the Thorne-Hartle model is not designed to describe directly the tidal deformations of a self-gravitating body, but only the backreaction on its external gravitational field. Backreactions to the interior field of the body are not accounted for by the model, which is why it can only be used to determine the effects of tidal heating if the overall structural changes of the body are sufficiently small and thus negligible.

Situations in which disregarding such structural changes no longer seems justified are those in which the shape, composition, and macroscopic thermal state of a given body (or collection of bodies) change drastically during gravitational interaction, e.g., when the body becomes heavily tidally deformed and heated during interaction with another, more massive celestial body, which may eventually lead to the collapse and disintegration of the body and its accretion by its much more massive companion. In such a situation, with both celestial bodies typically orbiting each other in circular motion, it is reasonable to expect that matter is constantly escaping from the system through the quasilocal surface of spacetime; at least as long as one assumes (inspired by Newton's theory of gravity) that the quasilocal surface represents the body's gravisphere, i.e., the spatially bounded sphere of influence of the collapsing body under consideration. Certainly also the collision of bodies-a process in which similarly strong tidal deformations can occur-cannot be described on the basis of a vacuum model like that of Hartle and Thorne.

It must be acknowledged, however, that describing the aforementioned phenomenological effects (even at the numerical level) is an extremely complicated venture; especially given the need to describe how the body's internal gravitational field changes such that the matter content of the spatially and temporally bounded gravitational system changes relative to its local physical environment.

Yet, despite the lack of a geometric model that could fully account for these effects, it shall be argued below that the integral expression (12) of the previous section can indeed be used to predict the behavior of very general types of matter distributions whose general form appears suitable 
to describe (at least in principle) processes in which objects are very strongly tidally deformed due to the phenomenological effects mentioned above. Moreover, it will be argued why and to what extent the Hartle-Thorne approximation proves insufficient to describe gravitational backreactions caused by isolated bodies that fail to be in almost perfect (macroscopic) internal thermal equilibrium.

As a basis for the arguments used, the theory of extended irreversible thermodynamics [21-26] shall be used at this point, with the main idea being that the corresponding physical framework is general enough to consider the case where tidal heating of a body (or collection of bodies) leads to the occurrence of heat flows and viscous stresses, and thus to non-negligible backreactions on the internal geometric field of the body (or bodies). In the mentioned theory, the matter distribution under consideration is that of a fluid mixture. Such a matter distribution can be fully characterized by three different quantities, i.e., the so-called thermal energy-momentum tensor $T^{a}{ }_{b}=\epsilon w^{a} w_{b}+\varpi^{a} w_{b}+$ $w^{a} \varpi_{b}+(p+\Pi) h_{b}^{a}+\Pi_{b}^{a}$, a particle number current $N_{A}^{a}=n_{A} w^{a}+\nu_{A}^{a}$, defined with respect to a number $A$ of species of particles, and a so-called covariant entropy current $S^{a}=s w^{a}+\eta^{a}$, where, in this context, the scalar fields $\epsilon, p$, and $s$ represent the energy density, pressure and total entropy density of the fluid mixture, $n_{A}$ is the particle density of a number of particle species $A$ and $\pi$ represents the viscous bulk pressure. The vector fields $w^{a}, \varpi^{a}, \nu_{A}^{a}$, and $\eta^{a}$ meanwhile represent the four-velocity vector of the system, an energy flux current and so-called particle diffusion and heat conduction fluxes, while the tensor field $\pi^{a}{ }_{b}$ represents the trace-free anisotropic viscous stresstensor. ${ }^{3}$ Depending crucially on whether one wishes to specify these quantities in the so-called energy or particle reference frames, different choices for some of the vector fields can typically be made, namely either $\nu_{A}^{a}=-\eta^{-1} q^{a}$, $\eta^{a}=\eta^{-1} \Theta q^{a}-Q^{a}$, and $p^{a}=0$ or $\nu_{A}^{a}=0, \eta^{a}=\beta q^{a}-Q^{a}$ and $p^{a}=q^{a}$, where $\beta=T^{-1} \equiv\left(\frac{\partial \varepsilon}{\partial s}\right)^{-1}$ is the inverse of the infamous Tolman-Ehrenfest temperature, $\eta$ is the relativistic enthalpy or "injection energy" per particle, $\Theta$ is the so-called thermal potential of the fluid mixture (relativistic chemical potential per particle and per temperature) and $Q^{a}$ is a second-order contribution which may be interpreted as a generalized heat flux and for which different approaches exist in the literature.

With respect to one of these choices, the physical behavior of fluid mixtures can be specified exactly in almost equilibrium states, in which the expressions given below must follow the differential laws

\footnotetext{
${ }^{3}$ As may be noted, all of these fields, including the thermal stress-energy tensor, could of course also be written down in $(3+1)$-form, but as will be made clear below, specifying these quantities in this form will prove unnecessary for the ensuing argument.
}

$$
\nabla_{a} T_{b}^{a}=0, \quad \nabla_{a} N_{A}^{a}=0, \quad \nabla_{a} S^{a} \geq 0
$$

The respective laws are the laws of local conservation of energy and particle number and the so-called ClausiusDuhem inequality, which is nothing more than the differential form of the entropy law of thermodynamics.

All this is important for the subject of tidal heating for the following reason: it makes a big difference for the form of the bulk part of the integral relation (12) whether the matter distribution under consideration is in thermal equilibrium with its environment or not. More precisely, it turns out that the bulk part of said relation is always zero (or, more precisely, can be chosen to be zero) for fluid mixtures that are in perfect thermal equilibrium, while this is not true for corresponding matter fields outside thermal equilibrium. For such matter distributions, as it turns out, the bulk part is always nonzero.

To see this, one may recall that the main equilibrium condition for relativistic viscous fluids in extended irreversible thermodynamics (as well as in classical theory) to be in thermal equilibrium is $\nabla_{a} S^{a}=0$; a condition that requires the complete absence of dissipative mechanisms. For this condition to be satisfied and for the corresponding system to actually reach a local thermostatic equilibrium state, the heat exchange between the fluids must come to a halt and the sum of all thermal potentials and chemical reaction rates must approach zero. Besides that, the thermal energy-momentum tensor and the particle number and entropy currents of the fluid mixture must be the same as that of an ordinary ideal fluid, meaning that $T^{a}{ }_{b}=$ $(\epsilon+p) w^{a} w_{b}+p \delta^{a}{ }_{b}, \quad N_{A}^{a}=n_{A} \cdot w^{a}, \quad$ and $\quad S^{a}=s \cdot w^{a}$. For this to hold, an equilibrium equation of state of the form $\epsilon+p=T\left(s+\sum_{A} \Theta_{A} n_{A}\right)$ must be valid, where each $\Theta_{A}$ represents a thermal potential associated with a species $A$ of particles (characterized in terms of the chemical potential $\mu_{A} \equiv \frac{\partial \epsilon}{\partial n_{A}}$ ). Ultimately, moreover, the motion of the fluids should be rigid in Born's sense, which means that the orthogonal distances between the adjacent material world lines of the two fluids must remain constant. In effect, this means that $h_{a}{ }^{c} h_{b}{ }^{d} \nabla_{(c} w_{d)}=0$ must hold, which in turn means that $\nabla_{(a} w_{b)}=0$ must be satisfied. However, from this it follows that the matter field under consideration must, at perfect thermal equilibrium, curve spacetime in such a way that a stationary gravitational field is generated with a timelike Killing vector field $\xi^{a}$ that is directly proportional to the four-velocity $w^{a}$, i.e., $\xi^{a}=\beta w^{a}$ with $\beta \equiv c \cdot N \equiv c \cdot \sqrt{-\xi_{a} \xi^{a}}$ with $c=$ const.

And while it is also possible in principle for internal thermal equilibrium to occur with respect to non-Killing observers, it is still reasonable to expect that the variables $\dot{N}, \dot{N}^{a}$, and $\dot{h}^{a b}$ for fluid mixtures in equilibrio are very close to zero (with respect to the Killing time parameter, which is admittedly the most natural choice). From this, in turn, it becomes clear that in nonequilibrium situations the 
bulk part of relation (12) can only be neglected and the Thorne-Hartle model can only be in agreement with the results of the quasilocal canonical framework of Brown and York if the $(3+1)$ quantities $\varepsilon, p_{a}$, and $S_{a b}$ introduced in the previous section are all exactly or at least close to zero.

For quasilocal surfaces, which are located very far from the boundary of the spatially finite internal gravitational field of the matter distribution under consideration, one can safely assume that the mentioned quantities actually become zero and thus for weak gravitational fields the Thorne-Hartle approximation remains valid and leads to correct results. An important reason for this is that dissipative heating of small, weakly gravitating bodies (such as, for example, Jupiter's satellite Io) it can be expected that only small amounts of electromagnetic radiation are emitted in the course of the tidal deformation process. However, for gravitational interactions of more massive objects, especially when a body is torn apart by tidal deformations or otherwise becomes unstable during the interaction period (with the consequence that not only the outer vacuum field of the body fluctuates, but also the inner field, which can lead to the emission of nonnegligible amounts of electromagnetic radiation), this can no longer be expected to be true, implying that the Hartle-Thorne approximation can no longer be used and it becomes necessary to consider integral relation (12) instead of relation (23). For certain types of matter accumulations, the structural changes in the gravitational source may even cause mass energy to flow out of the system at sometimes very high velocities (just think of relativistic jet flows in accreting supermassive black holes). Such phenomena are typically expected when bodies merge with each other; an effect that, like tidal heating, is described in various places in the literature based on the linearized approximation of general relativity [20,27-32].

Now, all this has an interesting consequence; the possibility of jointly measuring gravitational and electromagnetic radiation emitted during violent astrophysical events such as those described above suggests that the corrections calculated in the second part of the present work can lead to actual observable effects. More specifically, when considering Eq. (16), it becomes clear that if the Brown-York expression is to be a physically feasible candidate for gravitational mass energy not only in theory, but also in real life situations, there should be a shift in the total intensity when electromagnetic and gravitational radiation are emitted simultaneously from a gravitational matter source. This effect should then be measurable. Moreover, a comparable intensity shift should also occur when GEM effects are measured.

To illustrate this, the mass-energy inflow through the quasilocal surface of a spatially and temporally bounded gravitating system caused by GEM fields shall be calculated next. Using for this purpose. a perturbative splitting of the metric of the form (24) where the perturbation field $e_{a b}$ is used to define the trace-reversed gravitational potential $\psi_{a b}=e_{a b}-\frac{1}{2} \eta_{a b} e$, which is subject to the conditions $\partial^{a} \psi_{a b}=0,\left|\psi_{00}\right| \gg\left|\psi_{j k}\right|$, and $\left|\psi_{0 i}\right| \gg\left|\psi_{j k}\right|$, one finds that the linearized Einstein's equations reduce to the form

$$
\square \psi_{a b}=16 \pi T_{a b} .
$$

Here, it can be assumed for simplicity's sake that $T_{j k}=0$ for $j, k=1,2,3$ and therefore $S_{a b}$ applies. Moreover, it can be assumed that $s_{a b}=0$.

By defining next the fields $\psi_{00}(x) \equiv 2 \phi(x)$ and $\psi_{0 k}(x) \equiv 2 A_{k}(x)$, one finds that the linearized field equations read

$$
\square \phi=16 \pi \varepsilon, \quad \square A_{k}=16 \pi p_{k},
$$

where $k=1,2,3$ shall apply in the present context. The form of the solutions of these Poisson equations is

$$
\phi(t, \vec{x})=\int \frac{\phi(t-|\vec{x}-\vec{y}|, \vec{y})}{|\vec{x}-\vec{y}|} d^{4} y
$$

and

$$
\vec{A}(t, \vec{x})=\int \frac{\vec{A}(t-|\vec{x}-\vec{y}|, \vec{y})}{|\vec{x}-\vec{y}|} d^{4} y .
$$

The resulting line element of the metric reads

$d s^{2}=-(1-2 \phi) d t^{2}-4 A_{k} d t d x^{k}+(1+2 \phi) \delta_{j k} d x^{j} d x^{k}$

in Cartesian coordinates. The solutions obtained can be used to define gravielectric and gravimagnetic field strengths $\overrightarrow{\mathcal{E}}:=\frac{1}{2} \vec{\nabla} \phi$ and $\overrightarrow{\mathcal{B}}:=\vec{\nabla} \times \vec{A}$, which in turn can be used to set up the GEM pendants to the Maxwell equations and the Lorentz force law, i.e., the following set of relations

$$
\begin{aligned}
\vec{\nabla} \overrightarrow{\mathcal{E}} & =4 \pi \varepsilon, \\
\vec{\nabla} \overrightarrow{\mathcal{B}} & =0 \\
\vec{\nabla} \times \overrightarrow{\mathcal{E}} & =-\partial_{t} \overrightarrow{\mathcal{B}}, \\
\vec{\nabla} \times \overrightarrow{\mathcal{B}} & =-4 \pi \vec{p}+\partial_{t} \overrightarrow{\mathcal{E}}
\end{aligned}
$$

and

$$
\vec{F}=m(\overrightarrow{\mathcal{E}}+4 \vec{v} \times \overrightarrow{\mathcal{B}})
$$

which have to be met for the sake of consistency.

By considering the fact that $N=\sqrt{g^{t t}}=\sqrt{1+2 \phi} \approx$ $1+\phi$, one finds by neglecting higher-order terms that Eq. (12) takes the form 
$\frac{d E}{d t}=\int_{\Sigma_{t}}\left(\dot{\phi} \varepsilon-p_{k} \dot{A}^{k}\right) \omega_{h}-\int_{\Omega_{t}}\left(\lambda \dot{\phi} e-j_{k} \dot{\mathcal{A}}^{k}-\mathfrak{X}\right) \omega_{q}$,

where $\varepsilon=\frac{1}{8 \pi}\left(\overrightarrow{\mathcal{E}}^{2}+\overrightarrow{\mathcal{B}}^{2}\right)$ and $p_{k}=\frac{1}{\pi} \epsilon_{k i j} \mathcal{E}_{i} \mathcal{B}_{j}$ are the GEM energy current density and the GEM Poynting vector, and $\mathcal{A}^{b}=q^{b}{ }_{c} A^{c}$ is the vector potential on the quasilocal boundary surface. The crucial observation in this context is that the second corrective part $\mathfrak{X}_{2}$ of the integrand $\mathfrak{X}$ is different from zero. It reads

$$
\begin{aligned}
\mathfrak{X}_{2}= & -\lambda \eta(1+2 \phi) \varepsilon+\lambda^{2} \eta(1+2 \phi) p_{k} v^{k} \\
& +\lambda \eta(1+\phi) p_{k} \mathcal{A}^{k}-(1+2 \phi) p_{k} u^{k} .
\end{aligned}
$$

Imagining now that the two-surface $\Omega_{t}$ is a sphere at infinity, it becomes clear that the corresponding integral expression will also be different form zero, i.e.,

$$
\int_{\Omega_{t} \equiv S_{\infty}} \mathfrak{X}_{2} \omega_{q} \neq 0
$$

From this, however, it now follows that the quasilocal treatment of GEM effects requires consideration of the corrections resulting from the time derivative of the BrownYork mass given in the second section of this paper. Similar corrections should also arise, as mentioned earlier, when gravitational and electromagnetic radiation from a strongly gravitating matter source are measured cooperatively, since also in such a case a nonvanishing bulk-to-boundary energy inflow term should arise, and hence corrections to Einstein's quadrupole formula. The reason for this is that in both cases Einstein's field equations are satisfied for a nonvanishing matter source, which is why the correction terms resulting from the time derivative of the total quasilocal gravitational Hamiltonian cannot be neglected in either case.

From a practical point of view, the calculated corrections should manifest themselves in the form of a shift in the intensity of the measured radiation. It is therefore to be expected that the presence of a corresponding detectable intensity shift of incoming gravitational and electromagnetic radiation could provide a practical test of the utility and feasibility of the Brown-York expression as a reasonable candidate for the mass energy of the gravitational field.

\section{CONCLUSION}

In the present work, previous results by Booth and Creighton on quasilocal tidal heating were extended in that the mass-energy transfer through the quasilocal surface was calculated in a different manner, namely by varying the total gravitational Hamiltonian (bulk plus boundary parts) and not only the boundary part. As it turned out, all the results obtained are fully compatible with those of Booth and Creighton's work if it is assumed that the vacuum field equations of the theory are satisfied (as the authors actually did in their work). If this is not the case, however, as was shown, correction terms resulting from the time derivative of the Brown-York mass must be taken into account, one of which specifies how mass flows from the bulk into the boundary. To demonstrate the feasibility of the results obtained, their applicability to the geometric setting considered in Booth and Creighton's work on quasilocal tidal heating was demonstrated in the third section of the work and a comparatively simple geometric example was treated, namely, the mass-energy inflow through the quasilocal surface of a spatially and temporally bounded gravitating system caused by GEM fields. This simple example was used to show that the matter content of a spatially and temporally bounded gravitating system can change with time such that mass-energy flows through the quasilocal surface and thereby escapes from the system. From this insight, it was concluded that the obtained results can be used to describe tidal deformation and tidal heating effects in more challenging physical situations, such as in the description of accretion phenomena or merger processes in relativistic $N$-body systems.

\section{ACKNOWLEDGMENTS}

I want to greatly thank Ivan Booth for reading through and suggesting improvements to the first draft of the manuscript. I would also like to thank Friedrich Kupka for sharing his many insights into the phenomenology of astrophysics. Furthermore, I want to acknowledge the UAS Technikum Wien Library for financial support through its Open Access Funding Program as well as the Department Applied Mathematics and Physics for partially taking over the occurring fees.
[1] Ivan S. Booth and Jolien D. E. Creighton, Quasilocal calculation of tidal heating, Phys. Rev. D 62, 067503 (2000).

[2] Marc Favata, Energy localization invariance of tidal work in general relativity, Phys. Rev. D 63, 064013 (2001).

[3] Patricia Purdue, Gauge invariance of general relativistic tidal heating, Phys. Rev. D 60, 104054 (1999).
[4] Kip S. Thorne, Tidal stabilization of rigidly rotating, fully relativistic neutron stars, Phys. Rev. D 58, 124031 (1998).

[5] Richard Arnowitt, Stanley Deser, and Charles W. Misner, Republication of: The dynamics of general relativity, Gen. Relativ. Gravit. 40, 1997 (2008). 
[6] J. David Brown and James W. York Jr., Quasilocal energy and conserved charges derived from the gravitational action, Phys. Rev. D 47, 1407 (1993).

[7] Robert B. Mann, Donald Marolf, and Amitabh Virmani, Covariant counter-terms and conserved charges in asymptotically flat spacetimes, Classical Quantum Gravity 23, 6357 (2006).

[8] Hermann Bondi, M. G. J. Van der Burg, and A. W. K. Metzner, Gravitational waves in general relativity, VII. Waves from axi-symmetric isolated system, Proc. R. Soc. A. 269, 21 (1962).

[9] J. David Brown, Stephen R. Lau, and James W. York, Energy of isolated systems at retarded times as the null limit of quasilocal energy, Phys. Rev. D 55, 1977 (1997).

[10] Rainer K. Sachs, Gravitational waves in general relativity VIII. Waves in asymptotically flat space-time, Proc. R. Soc. A. 270, 103 (1962).

[11] A. Trautman, Gravitation: An Introduction to Current Research, edited by L. Witten (Wiley, New York, 1962).

[12] I. S. Booth and R. B. Mann, Moving observers, nonorthogonal boundaries, and quasilocal energy, Phys. Rev. D 59, 064021 (1999).

[13] Geoff Hayward, Gravitational action for spacetimes with nonsmooth boundaries, Phys. Rev. D 47, 3275 (1993).

[14] L. B. Szabados, Quasi-local energy-momentum and angular momentum in GR: A review article, Living Rev. Relativity 7, 4 (2004).

[15] James W. York Jr., Kinematics and dynamics of general relativity, in Sources of Gravitational Radiation (Cambridge University Press, 1979), pp. 83-126.

[16] James B. Hartle and Kip S. Thorne, Slowly rotating relativistic stars. II. models for neutron stars and supermassive stars, Astrophys. J. 153, 807 (1968).

[17] B. Leaute, The energy-momentum gravitational pseudotensor and Kerr-schild metrics, Gen. Relativ. Gravit. 7, 511 (1976).

[18] K. S. Virbhadra, Kerr-Newman metric and energy momentum pseudotensors of Einstein Tolman, Landau and Lifshitz, and Moller, Math. Today 9, 39 (1991).
[19] S. S. Xulu, Bergmann-Thomson energy-momentum complex for solutions more general than the Kerr-Schild class, Int. J. Theor. Phys. 46, 2915 (2007).

[20] Chia-Chen Chang, James M. Nester, and Chiang-Mei Chen, Pseudotensors and Quasilocal Energy-Momentum, Phys. Rev. Lett. 83, 1897 (1999).

[21] William A. Hiscock and Lee Lindblom, Stability and causality in dissipative relativistic fluids, Ann. Phys. (N.Y.) 151, 466 (1983).

[22] William A. Hiscock and Lee Lindblom, Generic instabilities in first-order dissipative relativistic fluid theories, Phys. Rev. D 31, 725 (1985).

[23] Werner Israel, Nonstationary irreversible thermodynamics: A causal relativistic theory, Ann. Phys. (N.Y.) 100, 310 (1976).

[24] Werner Israel and John M. Stewart, Transient relativistic thermodynamics and kinetic theory, Ann. Phys. (N.Y.) 118, 341 (1979).

[25] David Jou, Jose Casas-Vazquez, and Georgy Lebon, Extended irreversible thermodynamics revisited (1988-98), Rep. Prog. Phys. 62, 1035 (1999).

[26] L. D. Landau and E. M. Lifshitz, Theoretical physics, in Fluid Mechanics (Oxford, 1987), Vol. 6.

[27] Marek A. Abramowicz and P. Chris Fragile, Foundations of black hole accretion disk theory, Living Rev. Relativity 16, 1 (2013).

[28] Luc Blanchet, Gravitational radiation from post-newtonian sources and inspiralling compact binaries, Living Rev. Relativity 17, 2 (2014).

[29] Eric Burns, Neutron star mergers and how to study them, Living Rev. Relativity 23, 4 (2020).

[30] Ryan Hamerly and Yanbei Chen, Event horizon deformations in extreme mass-ratio black hole mergers, Phys. Rev. D 84, 124015 (2011).

[31] Uzair Hussain and Ivan Booth, Deformation of horizons during a merger, Classical Quantum Gravity 35, 015013 (2018).

[32] Gerhard Schäfer and Piotr Jaranowski, Hamiltonian formulation of general relativity and post-Newtonian dynamics of compact binaries, Living Rev. Relativity 21, 7 (2018). 